\title{
On Dirichlet Two-Point Boundary Value Problems for the Ermakov-Painlevé IV Equation
}

\author{
Pablo Amster and Colin Rogers
}

\begin{abstract}
Two-point boundary value problems of Dirichlet-type are investigated for a hybrid Ermakov-Painlevé IV equation. Existence and uniqueness results are established in terms of the Painlevé parameters. In addition, it is shown how Ermakov invariants may be used to systematically obtain solutions of a coupled Ermakov-Painlevé IV system in terms of seed solutions of the canonical integrable Painlevé IV equation.
\end{abstract}

\section{Introduction}

The six classical Painlevé equations, commonly denoted as PI-PVI, arise in a wide of physical applications and play a fundamental role in modern soliton theory (see e.g. Conte [1] and Clarkson [2] together with literature cited therein). In particular, Painlevé IV has important applications in hydrodynamics, nonlinear optics and quantum gravity theory. On the other hand, nonlinear coupled systems of Ermakov-Ray-Reid type [3-7] namely

$$
\begin{aligned}
& \ddot{\alpha}+\omega(t) \alpha=\frac{1}{\alpha^{2} \beta} \Phi(\beta / \alpha), \\
& \ddot{\beta}+\omega(t) \beta=\frac{1}{\alpha \beta^{2}} \Psi(\alpha / \beta),
\end{aligned}
$$

with their distinctive integral of motion

$$
\mathcal{I}=\frac{1}{2}(\alpha \dot{\beta}-\beta \dot{\alpha})^{2}+\int^{z=\beta / \alpha} \Phi(z) d z+\int^{w=\alpha / \beta} \Psi(w) d w
$$

likewise have extensive physical applications (see e.g. [8-14]). Just as solitonic systems and their associated classical Painlevé equations admit nonlinear superposition principles (permutability theorems) generated via invariance under Bäcklund transformations (see [1]), it is known that Ermakov-Ray-Reid systems also possess underlying nonlinear superposition laws, albeit of another kind [3,4]. Moreover, while solitonic and their associated Painlevé reductions generically admit linear representations, Ermakov-Ray-Reid systems likewise have been shown to have associated linear structure [15]. Despite these important commonalities investigations of Painlevé and Ermakov-Ray-Reid type systems have preceded independently until recently in [16] wherein hybrid Ermakov-Painlevé II symmetry reductions have been derived for N+1-dimensional resonant nonlinear Schrödinger systems.

A range of boundary value problems for the Painlevé II equation has been investigated in [17-21], notably in the context of multi-ion electrodiffusion. In the present work, a class of boundary value problems for a hybrid Ermakov-Painlevé IV equation is investigated. 
Moreover, in an appendix, an over-arching Ermakov-Painlevé IV system is set down and the key role of Ermakov invariants in its reduction to consideration of the canonical integrable Painlevé IV equation is established.

\section{The Class of Dirichlet Boundary Value Problems}

Here we consider the nonlinear 'Ermakov-Painlevé IV' equation [22]

$$
\Omega_{x x}-\left[\frac{3}{4} \Omega^{4}+2 x \Omega^{2}+x^{2}-\alpha\right] \Omega=\frac{\beta}{2 \Omega^{3}},
$$

which, on insertion of $\omega=\Omega^{2}$ leads to the canonical Painlevé IV equation (see e.g. $[1,2]$ ),

$$
\omega_{x x}=\frac{\omega_{x}^{2}}{2 \omega}+\frac{3}{2} \omega^{3}+4 x \omega^{2}+2\left(x^{2}-\alpha\right) \omega+\frac{\beta}{\omega},
$$

where $\alpha, \beta$ are Painlevé parameters.

In [23], Bassom et al considered a class of Dirichlet boundary value problems for the $\operatorname{avatar}(2.1)$ of the integrable Painlevé IV equation but with the specialisation $\beta=0$. In that paper, the concern was to isolate solutions $\Omega(\alpha: x)$ constrained by the boundary condition $\Omega(\infty)=0$. Bound state solutions were obtained via an integral equation formulation which, importantly, decay exponentially as $x=0$. Here, by contrast, our concern is with two-point boundary value problems wherein (2.1) with $\beta \neq 0$ are to be solved with Dirichlet conditions

$$
\Omega(0)=\Omega_{0}>0, \quad \Omega(1)=\Omega_{1}>0 .
$$

According to the standard terminology in singular problems, the cases $\beta<0$ and $\beta>0$ are termed, respectively, attractive and repulsive. In the first case, existence of classical solutions shall be proven; moreover, the solution is shown to be unique provided that the parameter $\alpha$ satisfies an appropriate smallness condition. For the repulsive case, we shall show that if one of the Dirichlet boundary values is prescribed, then at least one classical solution exists if and only if the other value belongs to a certain closed interval. Finally, we shall establish, for the attractive case, the existence of non-classical solutions that vanish over a prescribed finite set $B \subset(0,1)$. At each of these zeros, the left and right derivatives tend respectively to $-\infty$ and $+\infty$.

It is noted that a Ermakov-Painlevé IV type equation of the type (2.1) arises in the context of overarching two-component nonlinear systems as considered in the Appendix. Such systems may be obtained as particular similarity reductions of overarching coupled nonlinear Schrödinger systems. Dirichlet boundary conditions of the type (2.3) in that setting, correspond to prescribed 'total' squared amplitude type terms [22].

\section{Classical solutions}

\subsection{The attractive case $(\beta<0)$}

Theorem 3.1 The boundary value problem determined by (2.1) and (2.3) has a positive solution, which is unique if $\alpha \leq \lambda$, where $\lambda=10.15116403 \ldots$ is the first eigenvalue of the 
problem

$$
-u_{x x}+x^{2} u=\lambda u, \quad u(0)=u(1)=0 .
$$

Proof: Fix $M, \varepsilon>0$ such that $M>\Omega_{0}, \Omega_{1}>\varepsilon$ and

$$
\frac{3}{4} M^{5}-\alpha M+\frac{\beta}{2 M^{3}}>0, \quad \frac{3}{4} \varepsilon^{5}+2 \varepsilon^{3}+(1-\alpha) \varepsilon+\frac{\beta}{2 \varepsilon^{3}}<0 .
$$

From standard continuation methods, existence of solutions is guaranteed if the equation

$$
\Omega_{x x}=\sigma\left(\frac{3}{4} \Omega^{5}+2 x \Omega^{3}+\left(x^{2}-\alpha\right) \Omega+\frac{\beta}{2 \Omega^{3}}\right)
$$

with $0<\sigma \leq 1$ has no solutions satisfying (2.3) on the boundary of the set $\{\Omega \in C([0,1])$ : $\varepsilon<\Omega<M\}$. Indeed, if $\Omega$ solves (3.1) and achieves a maximum at some $x_{0} \in(0,1)$ with $\Omega\left(x_{0}\right)=M$, then $\Omega_{x x}\left(x_{0}\right)>0$, a contradiction. In the same way, it is shown that if $\Omega$ achieves a global minimum at $x_{0}$, then $\Omega\left(x_{0}\right) \neq \varepsilon$.

Now suppose that the boundary value problem comprising (2.1) with Dirichlet conditions (2.3) admits two different positive solutions and denote their difference by $\Omega$. It follows that $\Omega \Omega_{x x} \geq\left(x^{2}-\alpha\right) \Omega^{2}$, with the inequality being strict over a non-empty open subset of $(0,1)$. Thus,

$$
\int_{0}^{1}\left(\Omega_{x}^{2}+x^{2} \Omega^{2}\right) d x<\alpha \int_{0}^{1} \Omega^{2} d x \leq \frac{\alpha}{\lambda} \int_{0}^{1}\left(\Omega_{x}^{2}+x^{2} \Omega^{2}\right) d x
$$

a contradiction.

Remark 3.2 The exact value of $\lambda$ in the previous result can be computed numerically as the first positive zero of the equation

$$
{ }_{1} F_{1}\left(\frac{3-\lambda}{4}, \frac{3}{2}, 1\right)=0
$$

where ${ }_{1} F_{1}(a, b, z)=M(a, b, z)$ denotes the confluent hypergeometric function. Indeed, it is known that $y_{2}(a, z):=z e^{-z^{2} / 4}{ }_{1} F_{1}\left(\frac{2 a+3}{4}, \frac{3}{2}, \frac{z^{2}}{2}\right)$ solves the Weber equation

$$
y^{\prime \prime}(z)-\left(\frac{z^{2}}{4}+a\right) y(z)=0
$$

and hence $u(x):=2^{-1 / 2} y_{2}\left(-\frac{\lambda}{2}, \sqrt{2} x\right)$ verifies

$$
-u^{\prime \prime}(x)+x^{2} u(x)=\lambda u(x), \quad u(0)=0, u^{\prime}(0)=1 .
$$

Thus, the claim follows from the fact that $u(1)=2^{-1 / 2} y_{2}\left(-\frac{\lambda}{2}, \sqrt{2}\right)=e^{-1 / 2}{ }_{1} F_{1}\left(\frac{3-\lambda}{4}, \frac{3}{2}, 1\right)$. 


\subsection{The repulsive case $(\beta>0)$}

Theorem 3.3 For arbitrary $\Omega_{0}>0$, define

$$
I\left(\Omega_{0}\right):=\left\{\Omega_{1}>0: \text { (2.1), (2.3) has a positive solution }\right\} .
$$

Then $I\left(\Omega_{0}\right)=\emptyset$ or there exists $i\left(\Omega_{0}\right)>0$ such that $I\left(\Omega_{0}\right)=\left[i\left(\Omega_{0}\right),+\infty\right)$.

For a proof, we shall apply a shooting argument. For given $\lambda \in \mathbb{R}$, let $\Omega$ be the unique solution of (2.1) satisfying the initial conditions

$$
\Omega(0)=\Omega_{0}, \quad \Omega_{x}(0)=\lambda .
$$

Fix $M>\Omega_{0}, \Omega(1)$ such that $\frac{3}{4} M^{5}-\alpha M>0$ and define

$$
\phi_{M}(\lambda):=\left\{\begin{array}{cl}
M & \text { if } \Omega\left(x_{0}\right)=M \text { for some } x_{0}<1 \\
\Omega(1) & \text { otherwise. }
\end{array}\right.
$$

Lemma 3.4 $\phi_{M}: \mathbb{R} \rightarrow(0, M]$ is well defined and continuous. Furthermore, there exists $\lambda_{M}>0$ such that $\phi_{M}(\lambda)=M$ for $|\lambda| \geq \lambda_{M}$.

Proof:

Claim 1. If $\Omega$ is defined on $\left[0, x_{0}\right]$ and $\Omega\left(x_{0}\right)=M$, then $\Omega_{x}\left(x_{0}\right)>0$. Indeed, we may define $x_{M}=\inf \{x>0: \Omega(x)=M\}$, then $\Omega_{x}\left(x_{M}\right) \geq 0$ and $\Omega_{x x}\left(x_{M}\right)>0$. We deduce that $\Omega_{x}\left(x_{M}\right)>0$ and $\Omega$ is strictly increasing after $x_{M}$; in consequence, $x_{M}=x_{0}$.

Claim 2. There exists $c=c(\lambda)$ such that $\Omega(x) \geq c>0$ for all $x$. Indeed, if $\Omega>0$ on $\left[0, x_{0}\right)$ for some $x_{0}$, then by integration we obtain

$$
\Omega_{x}^{2}=\frac{\Omega^{6}}{4}+x \Omega^{4}-\int_{0}^{x} \Omega^{4}(s) d s+\left(x^{2}-\alpha\right) \Omega^{2}-2 \int_{0}^{x} s \Omega^{2}(s) d s-\frac{\beta}{2 \Omega^{2}}+C
$$

where $C=\lambda^{2}-\frac{\Omega_{0}^{6}}{4}+\alpha \Omega_{0}^{2}+\frac{\beta}{2 \Omega_{0}^{2}}$. If $\Omega$ tends to 0 as $x$ tends to $x_{0}$, then $\Omega_{x}^{2} \rightarrow-\infty$, a contradiction.

As a conclusion from the last claim, we deduce that if $\Omega$ does not reach the value $M$ before $x=1$, then it is defined on $[0,1]$ and hence $\phi_{M}$ is well defined and takes values in $(0, M]$. Continuity follows from the continuous dependence, together with the fact that $\Omega_{x}\left(x_{0}\right)>0$ when $\Omega\left(x_{0}\right)=M$.

Now suppose that $\Omega<M$ on $[0,1]$.

On the one hand, if $\lambda>0$, then from the fact that $\Omega_{x x}>-\alpha \Omega$ we deduce that $\Omega_{x}>\lambda-\alpha M$ and hence $\Omega>\Omega_{0}+(\lambda-\alpha M) x$. In particular, $\lambda<(1+\alpha) M-\Omega_{0}$. On the other hand, if $\lambda \ll 0$, then

$$
\Omega_{x}^{2}+\frac{\beta}{2 \Omega^{2}}=\lambda^{2}+o\left(\lambda^{2}\right)
$$


and taking $|\lambda|$ large enough we may assume that $\Omega_{x}^{2}+\frac{\beta}{2 \Omega^{2}}>\frac{\lambda^{2}}{2}$. Let

$$
A:=\left\{x \in[0,1]: \Omega(x) \geq \frac{2 \beta}{\lambda^{2}}\right\}
$$

and observe that, if $x \in A$, then $\Omega_{x}(x)^{2} \geq \frac{\lambda^{2}}{4}$. In particular, $A$ does not contain any critical point of $\Omega$.

Let $x_{1} \in A$ and assume that $\Omega_{x}<0$ on $\left[0, x_{1}\right]$. Then $\Omega_{x}<\frac{\lambda}{2}$ on $\left[0, x_{1}\right]$ and hence $\Omega\left(x_{1}\right)-\Omega_{0}<\frac{\lambda}{2} x_{1}$. We conclude that $x_{1}=O\left(|\lambda|^{-1}\right)$ and thus $\Omega$ must abandon the set $A$. Moreover, on any interval $(a, b) \subset[0,1] \backslash A$ it is seen that $\Omega^{2}<\frac{2 \beta}{\lambda^{2}}$ and thus $\Omega_{x x} \geq c|\lambda|^{3}$ for some constant $c>0$ independent of $\lambda$. It is deduced that $\Omega_{x}(b)-\Omega_{x}(a) \geq c|\lambda|^{3}(b-a)$. Since $\left|\Omega_{x}\right|=O(|\lambda|)$, we conclude that $b-a=O\left(|\lambda|^{-2}\right)$ and we deduce the existence of $x_{2}=O\left(|\lambda|^{-1}\right)$ such that $x_{2} \in A$ and $\Omega_{x}\left(x_{2}\right)>0$. Consequently, $\left[x_{2}, 1\right] \subset A$ and $\Omega_{x} \geq-\frac{\lambda}{2}$ for $x \geq x_{2}$. This implies that $\Omega(1)>\Omega\left(x_{2}\right)-\frac{\lambda}{2} \geq M$ if $\lambda \ll 0$, a contradiction.

\section{Proof of Theorem 3.3:}

Assume $I\left(\Omega_{0}\right) \neq \emptyset$, then there exists $\lambda$ such that the corresponding solution $\Omega$ is defined up to $x=1$. Set $M>\Omega_{0}, \Omega(1)$ as before and take $\lambda_{M}$ as in the previous lemma. We deduce that $\phi_{M}\left(\left[-\lambda_{M}, \lambda_{M}\right]\right)$ is a compact interval not containing 0 . Thus, $\operatorname{Im}\left(\phi_{M}\right)=\left[i\left(\Omega_{0}\right), M\right]$ for some $i\left(\Omega_{0}\right) \in(0, M)$ and the result follows since $M$ can be chosen arbitrarily large and $i\left(\Omega_{0}\right)$ does not change when $M$ is enlarged.

\section{Lacuna solutions}

Definition 4.5 A continuous function $\Omega:[0,1] \rightarrow \mathbb{R}$ satisfying (2.3) shall be called a (nonnegative) 'lacuna' solution of (2.1), (2.3) if there exists a nonempty finite set $B \subset(0,1)$ such that

1. $\Omega \equiv 0$ on $B$.

2. $\Omega>0$ and verifies $(2.1)$ on $(0,1) \backslash B$.

Remark 4.6 It shall be seen, as a consequence of the next theorem, that

$$
\lim _{x \rightarrow x_{*}^{+}} \Omega_{x}=-\lim _{x \rightarrow x_{*}^{-}} \Omega_{x}=+\infty
$$

for any $x_{*} \in B$.

Theorem 4.7 1. If $\beta>0$, then there are no lacuna solutions of (2.1), (2.3). 
2. Let $\beta<0$ and $0<x_{1}<\ldots<x_{N}<1$. Then there exists a lacuna solution $\Omega$ of (2.1), (2.3) such that $\Omega\left(x_{j}\right)=0$ for all $j$.

Proof: The first case follows from claim 2 in the preceding proof. For the attractive case $\overline{\beta<0}$, we shall divide the proof in several steps.

Step 1. There exists $\Omega$ positive solution of $(2.1)$ on $\left(0, x_{1}\right)$ such that $\Omega(0)=\Omega_{0}$, $\Omega\left(x_{1}\right)=0$. Indeed, set $M>\Omega_{0}$ large enough. As before, if $\Omega$ solves the initial value problem with $\lambda \gg 0$ then $\Omega$ reaches the value $M$ before $x=x_{1}$ and does not come down again. On the other hand, if $\Omega>0$ up to some $x_{*}<x_{1}$ and $\Omega(x) \rightarrow 0$ as $x \rightarrow x_{*}^{-}$, then it is seen that $\Omega_{x}(x) \rightarrow-\infty$ as $x \rightarrow x_{*}^{-}$. Thus, the mapping $\phi$ defined by

$$
\phi(\lambda)=\left\{\begin{array}{cl}
M & \text { if } \Omega(x)=M \text { for some } x<x_{1} \\
0 & \text { if } \Omega(x) \rightarrow 0 \text { as } x \rightarrow x_{*}^{-} \text {for some } x_{*}<x_{1} \\
\Omega\left(x_{1}\right) & \text { otherwise }
\end{array}\right.
$$

is well defined and continuous. Moreover, if $\lambda \ll 0$ then $\Omega_{x}^{2} \geq \lambda^{2}+o\left(\lambda^{2}\right)$ so we may assume that $\Omega_{x} \leq \frac{\lambda}{2}$; thus $\Omega(x) \leq \Omega_{0}+\frac{\lambda}{2} x$ and hence $\Omega$ vanishes before $x_{1}$. The claim follows from the continuous dependence on the initial conditions.

Analogously, we obtain:

Step 2. There exists $\Omega$ positive solution of $(2.1)$ on $\left(x_{N}, 1\right)$ such that $\Omega\left(x_{N}\right)=0$, $\Omega(1)=\Omega_{1}$.

Step 3. There exists $\varepsilon>0$ such that if $0<a<b<1$ and $b-a<\varepsilon$ then (2.1) has a solution $\Omega>0$ on $(a, b)$ with $\Omega(a)=\Omega(b)=0$. Indeed, following the ideas in [25] it suffices to prove that the autonomous problem

$$
\Omega_{x x}=\frac{3}{4} \Omega^{5}+2 \Omega^{3}+(1-\alpha)^{+} \Omega+\frac{\beta}{2 \Omega^{3}}, \quad \Omega(a)=\Omega(b)=0 .
$$

has a positive solution, which serves as a lower solution of the original problem. Multiplying (4.1) by $\Omega_{x}$ and setting $w:=\Omega^{2}$ the following equation is obtained:

$$
w_{x}^{2}=\left(R^{2}-w\right) T_{R}(w)
$$

where

$$
T_{R}(w):=-\frac{2 \beta}{R^{2}}-w\left(R^{4}+R^{2} w+w^{2}+4\left[R^{2}+w+(1-\alpha)^{+}\right]\right)
$$

and

$$
R=\max _{a \leq x \leq b} \Omega(x) .
$$

Thus, it is easily verified that (4.1) has a positive solution if and only if there exists $R>0$ such that

$$
\frac{3}{4} R^{8}+2 R^{6}+(1-\alpha)^{+} R^{4}+\frac{\beta}{2} \leq 0
$$


and

$$
\mathcal{I}(R):=\int_{0}^{R^{2}} \frac{d w}{\sqrt{\left(R^{2}-w\right) T_{R}(w)}}=\frac{b-a}{2} .
$$

Moreover, observe that $\mathcal{I}$ is continuous and

$$
\mathcal{I}(R) \leq \frac{1}{\sqrt{T_{R}\left(R^{2}\right)}} \int_{0}^{R^{2}} \frac{d w}{\sqrt{\left(R^{2}-w\right)}}=\frac{2 R}{\sqrt{T_{R}\left(R^{2}\right)}} \rightarrow 0
$$

as $R \rightarrow 0$. Hence, it suffices to take $\varepsilon=2 \mathcal{I}\left(R_{0}\right)$, where $R_{0}>0$ is the unique root of the left-hand side of (4.2).

\section{End of the proof}

Fix $\varepsilon>0$ as in step 3 and consider $x_{0}=y_{0}<\ldots<y_{M}=x_{N}$ such that $y_{j+1}-y_{j}<\varepsilon$ for $j=0, \ldots, M-1$ and $x_{i} \in B:=\left\{y_{j}: j=1, \ldots, M\right\}$ for all $i=0, \ldots, N$. A lacuna solution is constructed for this $B$ following the preceding steps.

\section{Appendix}

\section{A Coupled Ermakov-Painlevé IV System}

The importance of the Ermakov-Painlevé equation (1.1) as a canonical form is emphasised here by establishing its role in the construction of solution sets of an overarching coupled Ermakov-Painlevé IV system. It is remarked that Ermakov-Painlevé IV type systems have recently derived in [22] as symmetry reductions of coupled derivative nonlinear Schrödinger systems such as arise in plasma physics.

Here, we consider the hybrid two-component Ermakov-Painlevé IV system

$$
\begin{aligned}
& u_{x x}-\left[\frac{3}{4}\left(u^{2}+v^{2}\right)^{2}+2 x\left(u^{2}+v^{2}\right)+x^{2}-\alpha\right] u=\frac{\beta}{2 u^{3}}, \\
& v_{x x}-\left[\frac{3}{4}\left(u^{2}+v^{2}\right)^{2}+2 x\left(u^{2}+v^{2}\right)+x^{2}-\alpha\right] v=\frac{\beta}{2 v^{3}} .
\end{aligned}
$$

This is seen to admit a characteristic Ermakov invariant

$$
\left(u_{x} v-v_{x} u\right)^{2}+\frac{\beta}{2}\left[\left(\frac{u}{v}\right)^{2}+\left(\frac{v}{u}\right)^{2}\right]=\mathcal{E}
$$

together with the (non-local) Hamiltonian

$$
u_{x}^{2}+v_{x}^{2}-\frac{1}{4} \Sigma^{3}-2 \int x \Sigma d \Sigma-\int\left(x^{2}-\alpha\right) d \Sigma+\frac{\beta}{2}\left[\frac{1}{u^{2}}+\frac{1}{v^{2}}\right]=2 \mathcal{H} .
$$

where $\Sigma:=u^{2}+v^{2}$.

The identity

$$
\left(u^{2}+v^{2}\right)\left(u_{x}^{2}+v_{x}^{2}\right)-\left(u_{x} v-v_{x} u\right)^{2} \equiv\left(u u_{x}+v v_{x}\right)^{2}
$$


is now used together with the Ermakov and Hamiltonian invariant relations (A.2), (A.3) to show that

$$
\begin{aligned}
\Sigma[2 \mathcal{H} & \left.+\frac{1}{4} \Sigma^{3}+2 \int x \Sigma d \Sigma+\int\left(x^{2}-\alpha\right) d \Sigma-\frac{\beta}{2}\left(\frac{1}{u^{2}}+\frac{1}{v^{2}}\right)\right] \\
- & {\left[\mathcal{E}-\frac{\beta}{2}\left(\left(\frac{u}{v}\right)^{2}+\left(\frac{v}{u}\right)^{2}\right)\right]=\frac{1}{4}\left(\frac{d \Sigma}{d x}\right)^{2}, }
\end{aligned}
$$

whence,

$$
\Sigma\left[2 \mathcal{H}+\frac{1}{4} \Sigma^{3}+2 \int x \Sigma d \Sigma+\int\left(x^{2}-\alpha\right) d \Sigma\right]-\mathcal{E}-\beta=\frac{1}{4}\left(\frac{d \Sigma}{d x}\right)^{2} .
$$

The latter, in turn, leads to a Painlevé IV equation in $\Sigma$, namely

$$
\Sigma_{x x}=\frac{1}{2} \frac{\Sigma_{x}^{2}}{\Sigma}+\frac{3}{2} \Sigma^{3}+4 x \Sigma^{2}+2\left(x^{2}-\alpha\right) \Sigma+\frac{2}{\Sigma}(\mathcal{E}+\beta)
$$

which, in terms of $\Lambda=\Sigma^{1 / 2}$ produces the canonical Ermakov-Painlevé IV equation

$$
\Lambda_{x x}-\left[\frac{3}{4} \Lambda^{4}+2 x \Lambda^{2}+x^{2}-\alpha\right] \Lambda=\frac{\mathcal{E}+\beta}{\Lambda^{3}} .
$$

Importantly, the latter together with each in turn of the constituent nonlinear equations in the original Ermakov-Painlevé IV system (A.1) constitute Ermakov-Ray-Reid systems. This result in an additional pair of Ermakov-type invariant relations, viz

$$
\left(u_{x} \Lambda-\Lambda_{x} u\right)^{2}+(\mathcal{E}+\beta)\left(\frac{u}{\Lambda}\right)^{2}+\frac{\beta}{2}\left(\frac{\Lambda}{u}\right)^{2}=\mathbb{R}_{\mathrm{I}},
$$

and

$$
\left(v_{x} \Lambda-\Lambda_{x} v\right)^{2}+(\mathcal{E}+\beta)\left(\frac{v}{\Lambda}\right)^{2}+\frac{\beta}{2}\left(\frac{\Lambda}{v}\right)^{2}=\mathbb{R}_{\mathrm{II}} .
$$

Thus, on introduction of the new independent variable $\bar{x}$ according to

$$
d \bar{x}=\Lambda^{-2} d x
$$

and new dependent variables $U, V$ via

$$
U=\left(\frac{u}{\Lambda}\right)^{2}, \quad V=\left(\frac{v}{\Lambda}\right)^{2}
$$

it is seen that (A.9) and (A.10), in turn, yield

$$
d U^{1 / 2} / d \bar{x}= \pm \sqrt{\mathbb{R}_{\mathrm{I}}-(\mathcal{E}+\beta) U-\frac{\beta}{2} U^{-1}},
$$

and

$$
d V^{1 / 2} / d \bar{x}= \pm \sqrt{\mathbb{R}_{\mathrm{II}}-(\mathcal{E}+\beta) V-\frac{\beta}{2} V^{-1}}
$$


The latter pair show that, if $\mathcal{E}+\beta>0$ then

$$
U=\frac{1}{2(\mathcal{E}+\beta)}\left[\mathbb{R}_{\mathrm{I}}+\sqrt{\mathbb{R}_{\mathrm{I}}^{2}-2 \beta(\mathcal{E}+\beta)} \sin \left( \pm 2 \sqrt{\mathcal{E}+\beta} \bar{x}+\mathbb{K}_{\mathrm{I}}\right)\right]
$$

and

$$
V=\frac{1}{2(\mathcal{E}+\beta)}\left[\mathbb{R}_{\mathrm{II}}+\sqrt{\mathbb{R}_{\mathrm{II}}^{2}-2 \beta(\mathcal{E}+\beta)} \sin \left( \pm 2 \sqrt{\mathcal{E}+\beta} \bar{x}+\mathbb{K}_{\mathrm{II}}\right)\right]
$$

where $\mathbb{K}_{\mathrm{I}}$ and $\mathbb{K}_{\mathrm{II}}$ are constants of integration and it is required that $\mathbb{R}_{\mathrm{I}}^{2}>2 \beta(\mathcal{E}+\beta)$, $\mathbb{R}_{\text {II }}^{2}>2 \beta(\mathcal{E}+\beta)$. The relations (A.15), (A.16) are subject to the constraint $U+V=1$ and the latter relation may be used to calculate $V$ in terms of $U$ as given by (A.15) without recourse to (A.14). Moreover, addition of the integrals of motion (A.9), (A.10) together with use of the identity (A.4) and of the Ermakov invariant relation (A.2) is seen to impose the constraint

$$
\mathbb{R}_{\mathrm{I}}+\mathbb{R}_{\mathrm{II}}=2(\mathcal{E}+\beta) .
$$

In summary, solution pairs $\{u, v\}$ of the Ermakov-Painlevé IV system (A.1) may be constructed in terms of a seed solution $\Sigma$ of the canonical Painlevé IV equation (A.7) via relation

$$
u= \pm \sqrt{\frac{1}{2(\mathcal{E}+\beta)}\left(\mathbb{R}_{\mathrm{I}}+\sqrt{\mathbb{R}_{\mathrm{I}}^{2}-2 \beta(\mathcal{E}+\beta)} \sin \left( \pm 2 \sqrt{\mathcal{E}+\beta} \bar{x}+\mathbb{K}_{\mathrm{I}}\right)\right) \Sigma}
$$

together with

$$
v= \pm \sqrt{\Sigma-u^{2}}
$$

where $\bar{x}$ is obtained by integration of the relation (A.11), namely

$$
d \bar{x}=\Sigma^{-1} d x
$$

It is remarked that such seed solutions $\Sigma$ of Painlevé IV may be generated, in particular, via the application of established Bäcklund transformations (Bassom et al [24], Gromak [26]).

\section{Acknowledgement}

The authors thank Prof. D. Rial and the anonymous referee for the careful reading of the manuscript and their insightful remarks. This paper was partially supported by projects UBACyT 20020120100029BA and PIP 11220090100637 CONICET.

\section{References}

[1] R. Conte (Ed.), The Painlevé Property: One Century Later, Springer, New York (1999).

[2] P.A. Clarkson, Painlevé equations. Nonlinear Special Functions, J. Comput. Appl. Math. 153, 127-140 (2003). 
[3] J.R. Ray, Nonlinear superposition law for generalised Ermakov systems, Phys. Lett. A 78, 4-6 (1980).

[4] J.L. Reid and J.R. Ray, Ermakov systems, nonlinear superposition and solution of nonlinear equations of motion J. Math. Phys. 21, 1583-1587 (1980).

[5] C. Rogers, C. Hoenselaers and J.R. Ray, On 2+1-dimensional Ermakov systems, J. Phys. A: Math. Gen. 26, 2625-2633 (1993).

[6] C. Rogers and W.K. Schief, Multi-component Ermakov systems: structure and linearization, J. Math. Anal. Appl. 198, 194-220 (1996).

[7] W.K. Schief, C. Rogers and A.P. Bassom, Ermakov systems with arbitrary order and dimension: structure and linearization, J. Phys. A: Math. Gen. 29, 903-911 (1996).

[8] A.M. Goncharenko, Y.A. Logvin, A.M. Samson, P.S. Shapovalov and S.I. Turovets, Ermakov Hamiltonian systems in nonlinear optics of elliptic Gaussian beams, Phys. Lett. A 160, 138-142 (1991).

[9] C. Rogers and H. An, Ermakov-Ray-Reid systems in 2+1-dimensional rotating shallow water theory, Stud. Appl. Math. 125, 275-299 (2010).

[10] C. Rogers, B. Malomed, K.W. Chow and H. An, Ermakov-Ray-Reid systems in nonlinear optics, J. Phys. A: Math. \& Theor. 43, 455214 (2010).

[11] C. Rogers and W.K. Schief, The pulsrodon in 2+1-dimensional magnetogasdynamics. Hamiltonian-Ermakov reduction, J. Math. Phys. 52, 083701 (2011).

[12] C. Rogers and W.K. Schief, On the integrability of a Hamiltonian reduction of a 2+1dimensional non-isothermal rotating gas cloud system, Nonlinearity 24, 3165-3178 (2011).

[13] C. Rogers, B. Malomed and H. An, Ermakov-Ray-Reid reductions of variational approximations in nonlinear optics, Stud. Appl. Math. 129, 389-413 (2012).

[14] W.K. Schief, H. An and C. Rogers, Universal and integrable aspects of an elliptic vortex representation in 2+1-dimensional magnetogasdynamics, Stud. Appl. Math. 130, 49-79 (2013).

[15] C. Athorne, C. Rogers, U. Ramgulam and A. Osbaldestin, On linearisation of the Ermakov system, Phys. Lett. A 143, 207-212 (1990).

[16] C. Rogers, On a coupled nonlinear Schrödinger system. A Ermakov connection, published online Stud. Appl. Math. (2014).

[17] M.C. Mariani, P. Amster and C. Rogers, Dirichlet and periodic-type boundary value problems for Painlevé II, J. Math. Anal. Appl. 265, 1-11 (2002).

[18] P. Amster, M.C. Mariani, C. Rogers and C.C. Tisdell, On two-point boundary value problems in multi-ion electrodiffusion, J. Math. Anal. Appl. 289, 712-721 (2004). 
[19] P. Amster, M.K. Kwong and C. Rogers, On a Neumann boundary value problem for the Painlevé II equation in two-ion electrodiffusion, Nonlinear Analysis 74, 2897-2907 (2011).

[20] P. Amster, M.K. Kwong and C. Rogers, A Neumann boundary value problem in two-ion electrodiffusion with unequal valencies, Discrete and Continuous Dynamical Systems 17, 2299-2311 (2012).

[21] P. Amster, M.K. Kwong and C. Rogers, A Painlevé II model in two-ion electrodiffusion with radiation boundary conditions, Nonlinear Analysis: Real World Applications 16, 120-131 (2014).

[22] C. Rogers, Hybrid Ermakov-Painlevé IV systems, submitted (2014).

[23] A.P. Bassom, P.A. Clarkson, A.C. Hicks and J.B. Mcleod, Integral relations and exact solutions for the fourth Painlevé equation, Proc. Roy. Soc. Lond. A 437, 1-24 (1992).

[24] A.P. Bassom, P.A. Clarkson and A.C. Hicks, Bäcklund transformations and solution hierarchies for the fourth Painlevé equation, Stud. Appl. Math. 95, 1-71 (1995).

[25] P. Amster and C. Rogers, On a Ermakov-Painlevé II reduction in three-ion electrodiffusion. A Dirichlet boundary value problem, submitted (2014).

[26] V.I. Gromak, Bäcklund transformations of Painlevé equations and their applications, in The Painlevé Property. One Century Later (Ed. R. Conte) CRM Series in Mathematical Physics, Springer, New York pp 687-734 (1999). 